\title{
Correction of B-scan distortion for optimum ultrasonic imaging of backwalls with complex geometries
}

\author{
S Davì, C Mineo, C MacLeod, S G Pierce, A Gachagan, S Paton, G Munro, J O'Brien-O'Reilly and C McCubbin
}

\begin{abstract}
Ultrasound undergoes refraction and reflection at interfaces between media of different acoustic refractive indices. The most common ultrasonic method (pulse-echo) monitors the reflected energy to infer the presence of flaws, whereas the lower amplitude of refracted signals is ignored. When the reflector is orientated normally with respect to the ultrasonic beam, the received echo signal shows the maximum amplitude. The pulse-echo method also relies on monitoring the amplitude of the backwall echo to identify or confirm the presence of defects. This works well for parts with constant thickness and with planar backwalls. Unfortunately, parts with complex backwalls are common to many industrial sectors. For example, applications such as aerospace structures often require parts with complex shapes. Assessing such parts reliably is not trivial and can cause severe downtime in the aerospace manufacturing processes or during in-service inspections. This work aims to improve the ultrasonic inspectability of parts with complex backwalls, through sending ultrasonic beams from the frontwall side. Ultrasonic phased array probes and state-of-the-art instrumentation allow ultrasonic energy to be sent into a part at wide ranges of focusing depths and steering angles. This allows for tracking of the backwall profile, thus hitting it normally and maximising the amplitude of the reflected echo at any point. However, this work has shown that a cross-sectional scan resulting from multiple ultrasonic beams, which are sent at variable incidence angles, can present significant geometrical distortion and cannot be of much use for accurate defect visualisation and sizing. This paper introduces a generalised algorithm developed to remove geometric distortions and the effect that variable refraction coefficients have on the transmitted and received amplitudes. The algorithm was validated through CIVA simulations for two example parts with complex backwalls, considering isotropic materials.
\end{abstract}

Keywords: ultrasonic imaging, complex geometries, data visualisation, B-scans.

\section{Introduction}

\subsection{Ultrasonic imaging of complex geometries}

Ultrasonic imaging has become the most common technique for nondestructive testing (NDT) of solid structures ${ }^{[1]}$. Using ultrasound for testing purposes has significant advantages, including: high penetration for a wide range of materials; the capability to estimate the size, orientation, shape and nature of defects; and the capability to evaluate the structure of alloys of components with different acoustic properties ${ }^{[2]}$. Moreover, ultrasonic NDT methods are nonhazardous to operations or to nearby personnel and have no effect on equipment and materials in the vicinity. Ultrasonic testing is also easy to deploy through portable equipment or highly automated approaches for large structures.

However, ultrasonic energy is strongly attenuated by air gaps, due to the low acoustic impedance of air. A suitable coupling medium is needed to transfer the ultrasonic waves from the transducer to the material undergoing inspection. Coupling is often achieved by immersion of both the transducer and the specimen in a body of liquid or by actual contact through a thin film of liquid. The ultrasonic vibrations pass through the coupling medium and are reflected by any discontinuities that may be encountered. The reflected energy is received by the same or by a different transducer and is converted into an electrical signal. Imaging the profile of a complex-geometry part through a rigid linear array transducer can prove problematic (where direct contact is not always possible). Therefore, this is overcome by coupling through an intermediate medium such as a Perspex shoe or a fluid, as in the case of immersion testing. However, the computational complexity involved in imaging through a dual media makes real-time inspection difficult, as variations in geometry directly influence the imaging algorithms. Considering Fermat's principle, an ultrasonic ray starting at a given point arrives at another point following the lowest time-offlight path. As a consequence, in the presence of a curved interface separating two media with different sound velocities, the lowest time-of-flight path can diverge from a single straight segment ${ }^{[3]}$. Since the introduction of advanced ultrasonic data acquisition and imaging techniques, such as full matrix capture (FMC) and the total focusing method (TFM) $)^{[4]}$, imaging through a non-planar surface has become a time-intensive task ${ }^{[5]}$, where it is necessary to calculate the time-of-flight from each transmit/receive element combination to a given pixel in the region of interest through the refractive

\section{Submitted 27.09.19/ Accepted 27.02.20}

Santi Davì, Carmelo Mineo, Charles MacLeod, Gareth Pierce* and Anthony Gachagan are with the Centre for Ultrasonic Engineering (CUE), Department of Electronic \& Electrical Engineering, University of Strathclyde, Royal College Building, 204 George Street, Glasgow, UK.

Scott Paton, Gavin Munro, Janet O'Brien-O'Reilly and Coreen McCubbin are with Spirit AeroSystems (Europe) Limited, Prestwick, UK.

*Corresponding author. Tel: +44 (0)141548 2617 (office) / +44 (0)7970 816426 (work) / +44 (0)7966 967522 (mobile); Email:s.g.pierce@strath.ac.uk 
boundary. Extensive investigation has been undertaken in the efficient imaging of such data in post-processing, due to the large amount of data for data storage ${ }^{[6-8]}$ and in real-time inspection ${ }^{[5]}$. However, the large datasets associated with FMC and the number of calculations required to effectively image ultrasonic data for a complex geometry still limit the scanning speed rates ${ }^{[9]}$. This is a bottleneck in the production of large, high-value and safety-critical structures, where robust assessment of structural integrity is an unavoidable requirement.

\subsection{The need to increase speed and improve part inspectability}

To meet future demand projections (for example in the aerospace sector) and overcome the bottlenecks of safety-critical NDT inspections, new automated scanning systems have been developed by a variety of research and development teams ${ }^{[10-13]}$. Producing reliable automated solutions has become an industry priority to drive down inspection times and ensure repeatability. Applications of six-axis robotic arms in the NDT field have been published and there is a growing interest in using such automation solutions ${ }^{[14-18]}$. In aerospace transportation, the need to reduce operational costs and $\mathrm{CO}_{2}$ emissions into the environment is leading to aircraft that are more efficient ${ }^{[19]}$. Design engineers are focusing on reducing the structural weights by using innovative materials and geometries. Research has enabled the generation of optimum robotic tool-paths to inspect curved parts ${ }^{[20,21]}$, the introduction of suitable ultrasonic coupling techniques (for example purposely developed water-jet nozzles ${ }^{[17]}$ and rolling water chambers ${ }^{[2]}$ ) and high data transfer rates. State-of-the-art ultrasonic phased array instrumentation and fast communication sockets have enabled component scanning rates of up to $117.6 \mathrm{~m}^{2} / \mathrm{h}$ with a robotically manipulated $5 \mathrm{MHz}$ 64-element (1 mm pitch) phased array transducer, when acquiring frames (B-scans) at $1 \mathrm{~mm}$ intervals and firing all probe elements at once (thus using an unfocused ultrasonic beam) ${ }^{[23]}$. The scanning rate of such systems drops to $25 \mathrm{~m}^{2} / \mathrm{h}$ when using traditional beamforming with an eight-element sub-aperture. Indeed, 57 firings are required to sweep the ultrasonic beam through the phased array probe and the maximum robotic manipulation speed is limited to $150 \mathrm{~mm} / \mathrm{s}$. The figures are worse for phased array FMC; it has typically not yet been used for the robotic inspection of large structures due to the low number of frames per second (FPS) that can currently be achieved. Real-time FMC imaging is currently performed at up to $40 \mathrm{FPS}^{[5]}$, which would limit the scanning rate to approximately $6.5 \mathrm{~m}^{2} / \mathrm{h}$.

Therefore, although research and technology advancement will speed up FMC and TFM imaging in the future, automated ultrasonic phased array inspection is to date mostly performed through traditional beamforming or the emerging multi-aperture excitation $\operatorname{mode}^{[24]}$. These data acquisition approaches work very well for imaging all part regions that present constant or gently changing thicknesses, as in such scenarios a strong backwall reflection is received. Conversely, regions with severe backwall tapering or with complex backwalls (for example radii) are notoriously challenging to inspect, since the backwall reflects the ultrasonic energy at high angles with respect to the incident beam and the received echo is often not received or strongly attenuated. Previous work has demonstrated the possibility to inspect the radii of structural stiffening stringers and ribs using concave probes, placing them coaxially to the surfaces of radii, when inspecting from the radii side, or phased array probes defining appropriate delay laws ${ }^{[21,25-27]}$. Although this approach maximises the ultrasonic energy that enters the part, it does not change the fact that the backwall reflection is often impossible to receive. Moreover, the quality of such an inspection is strongly dependent on the accuracy of the geometric alignment between the probe and the centre of curvature of the radii. Furthermore, the side of the part with complex geometry is often not accessible, especially for in-service components, and can present restricted areas where probe placement is difficult.

This paper focuses on improving the ultrasonic inspectability of parts with complex backwalls. Progress in this direction is being achieved by modelling of ultrasonic wave propagation in such parts. Promising results are possible through the use of state-of-the-art instrumentation that allows complex focal laws to be exploited to send ultrasonic energy into a part at wide ranges of focal depths and steering angles. This allows for tracking of the backwall profile, thus allowing normal angles of incidence to be achieved and maximising the amplitude of the reflected echo at any point. This paper presents a new algorithmic approach to improve the visualisation of inspection results relative to regions with complex backwalls. Ultrasonic data can be collected and displayed in a number of different formats. Previous work has highlighted the importance of introducing new data visualisation and analysis tools capable of mapping complex geometries and enabling easy detection of defects ${ }^{[12,28-31]}$.

B-scans resulting from multiple A-scans, which originate from ultrasonic beams sent at variable incidence angles, can present significant geometrical distortion and cannot be directly used for accurate defect visualisation and sizing. Therefore, this paper introduces a generalised algorithm developed to remove geometric distortions and the effect that variable refraction coefficients have on the transmitted and received amplitudes. This work has focused on isotropic materials as a proof-of-concept phase.

\section{Review of current visualisation approaches}

Strictly speaking, the B-scan presentation refers to the image produced when the data collected from an ultrasonic inspection is plotted on a cross-sectional view of the component. The original definition, according to BS EN 1330-4:2000 'Non-destructive testing - Terminology - Terms used in ultrasonic testing', is the 'image of the results of an ultrasonic examination showing a crosssection of the test object perpendicular to the scanning surface and parallel to a reference direction'. The cross-section is the plane through which the individual A-scans have been collected. Therefore, the B-scan will generally be the plane that contains the ultrasonic beam axes.

Phased array probes use a range of elements, all individually wired, pulsed and time-shifted on both pulsing and receiving. In the traditional beamforming scanning mode, the elements are typically pulsed in groups (known as sub-apertures). With userfriendly systems, a typical set-up calculates the time delays from operator input or uses a predefined file calculated for the inspection angle, focal distance and scan pattern ${ }^{[32]}$. The time delay values are back-calculated using time-of-flight from the focal spot and the scan is assembled from individual 'focal laws'. Time delay circuits use high-resolution clocks (with nanosecond resolution) to allow for the pulsing and receiving of each element at the required time.

Phased array electronic pulsing and receiving have introduced significant opportunities for a variety of scan patterns. Multiplexing along an array generates electronic scans (E-scans) that permit rapid coverage with a tight focal spot. If the array is flat and linear, then the scan pattern is a simple B-scan. A sectorial scan (S-scan) refers to either the beam movement or the data display. As a data display, the $\mathrm{S}$-scan is a $2 \mathrm{D}$ view of all A-scans from a specific set of elements 
corrected for delay and refracted angle. When used in relation to the beam movement, it refers to the set of focal laws that sweep through a defined range of angles using the same set of elements. One application for S-scans involves a stationary array sweeping across a relatively inaccessible component, such as a welded area, to map out the features and defects. Dynamic depth focusing is also a beamforming technique that allows the spatial response of the array to be changed by moving the focal spot of the probe ${ }^{[33-35]}$.

Sizing a defect detected through a simple B-scan and an S-scan is straightforward. However, if a curved array is used to perform an electronic scan of a corner (see Figure 1), then the defect size in the index axis cannot be directly obtained from the B-scan but must be calculated. The real size $(s)$ of a defect in the index axis is a function of the apparent size $(C)$, as shown in the B-scan, the radius $(r)$ and the thickness $(t)$ of the corner, the radius of the probe $(R)$ and the depth $(d)$ of the defect below the surface. The reason for this is that the rectified cross-sectional view of the corner, represented in the resulting B-scan, inevitably distorts the appearance of any ultrasonic reflectors found within the material. Equations (1) and (2) show the formulae for the inner diameter (ID) and outer diameter (OD) inspection approaches, respectively ${ }^{[36]}$ :

$$
\begin{gathered}
s=\frac{C \cdot(r+d)}{R} \cdots . \\
s=\frac{C \cdot(r+t-d)}{R}
\end{gathered}
$$

Equations (1) and (2) are simple algebraic formulae that an NDT inspector can easily apply every time accurate sizing is required for each indication detected through radius phased array probes. The simplicity of such formulae to correct the geometrical distortion is due to the specific scenario, where the cylindrical probe aperture is positioned coaxially to the surface of the corner. In this case, all ultrasonic beam axes are perpendicular to the surface of the probe and hit the surface of the specimen perpendicularly too. However, simple correction formulae are not available for complex situations. The remaining part of this paper introduces a generalised algorithm to remove the geometrical distortions present in B-scans, which are constituted by a sequence of A-scans relative to ultrasonic beams that penetrate the part with variable incident angles.

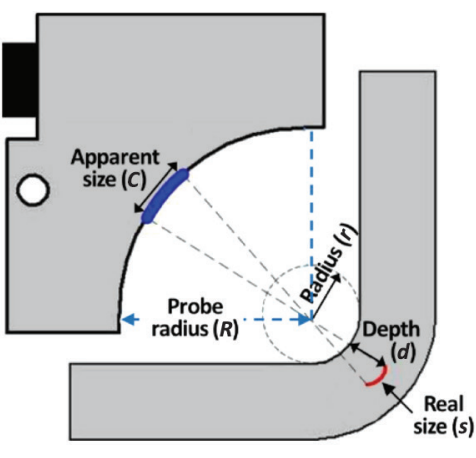

(a)

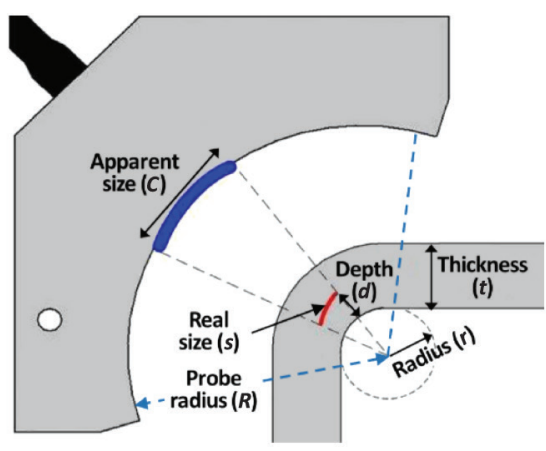

(b)

Figure 1. Curved array performing an electronic scan of a corner with: (a) ID inspection approach; and (b) OD inspection approach ${ }^{[36]}$

\section{B-scan correction algorithm}

When a longitudinal ultrasonic wave strikes an interface between two different media, specific phenomena are observed. The wave is both reflected and refracted. If the second medium is solid, a phenomenon known as mode conversion also causes the refracted ultrasonic energy to decompose into two waves: a shear wave $(S)$ and a longitudinal wave $(L)$ component. This work ignores the refracted shear wave, since it has low-energy content for the range of incident angles considered. Therefore, the original incident longitudinal beam is reflected and refracted by the interface between the two media, as is explained well by Fermat's principle developed in optics ${ }^{[37]}$ and by Snell's Law in acoustics $^{[38]}$.

Snell's Law describes, for isotropic materials, the relationship between the angles and the velocities of the waves. Snell's Law equates the ratio of material velocities $\left(V_{1 L}, V_{2 L}\right.$ and $\left.V_{2 S}\right)$ to the ratio of the sine of the angles $\left(\theta_{1 L}, \theta_{2 L}\right.$ and $\left.\theta_{2 S}\right)$ the wave propagation directions form with the normal to the interface at the incidence point, as shown in Equation (3):

$$
\frac{\sin \theta_{1 L}}{V_{1 L}}=\frac{\sin \theta_{2 L}}{V_{2 L}}=\frac{\sin \theta_{2 S}}{V_{2 S}}
$$

If unitary intensity is assigned to the wave striking the interface between medium 1 and medium 2 with incidence angle $\theta_{1 L}$, refraction angles of longitudinal and shear waves equal to $\theta_{2 L}$ and $\theta_{2 S}$, respectively, are produced. The intensity of the reflected and refracted (transmitted) longitudinal waves, respectively indicated by $I_{12}^{R}$ and $I_{12}^{T}$, can be computed using Equations (4) and (5):

$$
I_{12}^{R}=\left(\frac{Z_{L} \cos ^{2} 2 \theta_{2 S}+Z_{S} \sin ^{2} 2 \theta_{2 S}-Z_{1}}{Z_{L} \cos ^{2} 2 \theta_{2 S}+Z_{S} \sin ^{2} 2 \theta_{2 S}+Z_{1}}\right)^{2}
$$

$$
I_{12}^{T}=\frac{\rho_{1}}{\rho_{2}} \frac{\tan \theta_{1 L}}{\tan \theta_{2 L}}\left(\frac{2 Z_{L} \cos 2 \theta_{2 S}}{Z_{L} \cos ^{2} 2 \theta_{2 S}+Z_{S} \sin ^{2} 2 \theta_{2 S}+Z_{1}}\right)^{2} \ldots
$$

where $Z_{1}=\rho_{1} V_{1 L} / \cos \theta_{1 L}, Z_{L}=\rho_{2} V_{2 L} / \cos \theta_{2 L}$ and $Z_{S}=\rho_{2} V_{2 S} / \cos \theta_{2 S}$, with $\rho_{1}$ and $\rho_{2}$ indicating the material densities ${ }^{[39]}$.

Reflection, refraction, mode conversion and energy attenuation take place at each interface encountered between different media. When inspecting an isotropic and homogeneous part in ultrasonic pulse-echo mode using the immersion technique, the ultrasonic beam encounters three interfaces during its path. After being generated by the transducer, the beam travels through water until striking the interface between the water and the front surface of the part (the frontwall), where part of the beam energy is refracted within the material of the part. The refracted beam continues its path within the volume of the part until it hits the interface at the other side of the part (the backwall), where part of the refracted beam energy is reflected, creating what is called 'echo'. Then, the echo travels back to the frontwall interface and the wave component, which is refracted at this interface, arrives back at the transducer surface and is finally converted into a measurable signal. Therefore, the refraction and reflection angles and the energy attenuation at every interface must be considered, through Equations (3)-(5), to verify the inspectability of a part with a given cross-sectional geometry.

Figure 2 shows the interactions between the ultrasonic longitudinal wave generated by a single-element transducer (or by a single aperture in a phased array probe) and the interfaces of a solid part immersed in water. In Figures 2(a) and 2(b), the incident 
longitudinal wave $\left(L_{1}\right)$ strikes the first interface between the water and the solid perpendicularly; part of its energy goes to the reflected wave $\left(L_{1}^{R}\right)$ and the residual part goes to the transmitted wave $\left(L_{1}^{T}\right)$. Part of the energy of $L_{1}^{T}$ gets reflected at the backwall and forms the echo wave $\left(L_{E}\right)$. In Figure 2(a), $L_{E}$ returns to the first interface and strikes it perpendicularly, producing the transmitted echo wave $\left(L_{E}^{T}\right)$ that reaches the transducer. Conversely, the complex backwall geometry in Figure 2(b) does not allow the backwall echo to return to the transducer, since $L_{E}$ strikes the interface at an angle and the resulting wave refracted into water misses the receiving probe surface. In Figure 2(c), for any point along the backwall and keeping constant the length (W) of the segment the incident wave travels in water, the probe is tilted by an angle. Such an angle, calculated through Snell's Law, produces a refracted longitudinal wave that arrives perpendicularly to the backwall. Then, $L_{1}^{T}$ is optimally reflected by the backwall interface to form $L_{E}$, which follows the same path of the incident wave in reverse. In this case, the amplitude (A) of $L_{E}^{T}$ is always lower than the amplitude of the wave received in Figure 2(a), because of the mode conversion at the first interface that disperses part of the energy into the shear wave $\left(S_{1}^{T}\right)$. However, the approach in Figure 2(c) allows the amplitude of the echo, receivable from any point along the complex backwall, to be maximised when using the immersion pulse-echo inspection technique.

This work considers the approach represented in Figure 2(c) to map the backwall geometry. CIVA software ${ }^{[40]}$ has been used to simulate the beam propagation in an aluminium sample. The water immersion pulse-echo technique was simulated, with $V_{1 L}=1483 \mathrm{~m} / \mathrm{s}$ being set for the acoustic wave propagation velocity in water. The water path was set equal to $\mathrm{W}=10 \mathrm{~mm}$, in order to keep the near field of the probe $(\sim 7.6 \mathrm{~mm})$ above the front surface of the part. Velocities equal to $V_{2 L}=6360 \mathrm{~m} / \mathrm{s}$ and $V_{2 S}=3100 \mathrm{~m} / \mathrm{s}$ were respectively set for the longitudinal and shear wave propagation speeds in aluminium. A cylindrical $3 \mathrm{~mm}$-diameter single-element probe, with a flat active surface and a central frequency of $5 \mathrm{MHz}$, was chosen for the CIVA simulations. The simulated A-scans were imported and processed in MATLAB ${ }^{\circ}$. The ultrasonic beams produced by a single-element probe (in pulse-echo configuration) were simulated. The probe positions and orientations were calculated appropriately, in accordance with Snell's Law, in order to ensure that the refracted wave $\left(L_{1}^{T}\right)$, relative to each beam, strikes the backwall perpendicularly. Figure 3 shows a diagram reporting the problem tackled by this work. The area enclosed within the dotted rectangle is described in the remaining part of this paper.

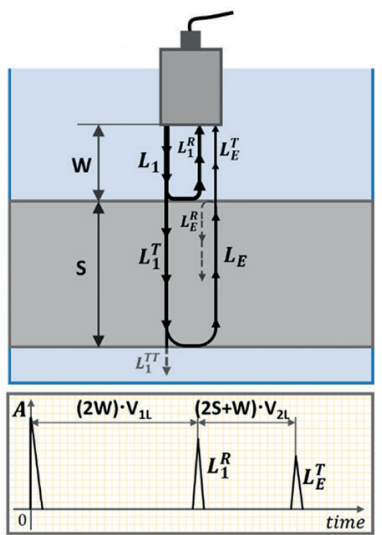

(a)

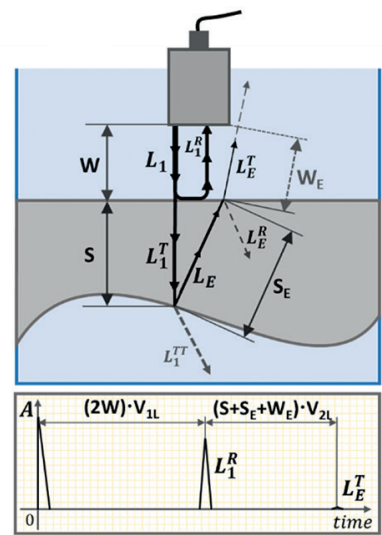

(b)

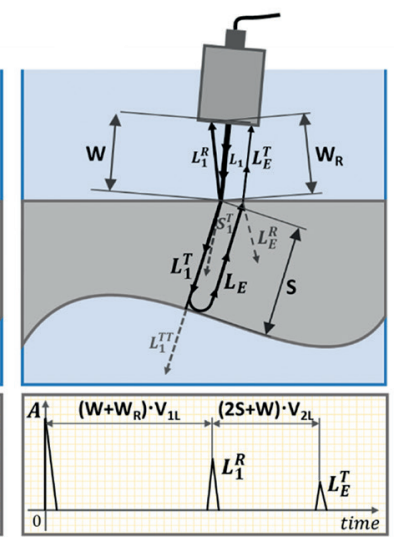

(c)
Figure 2. Ultrasonic pulse-echo for: (a) simple; and (b) and (c) complex backwalls
Problem: Ultrasonic mapping of complex backwall and display of undistorted B-scan

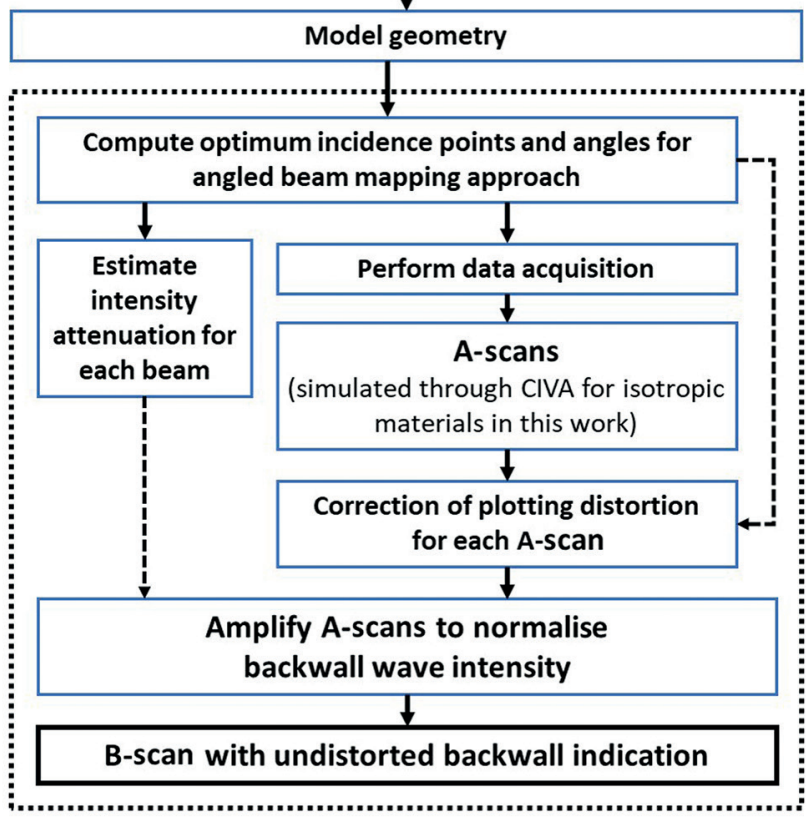

Figure 3. The problem tackled by this work

\subsection{Geometric correction}

The sample geometry, given in Figure 4, was imported in CIVA. It has a flat front surface and a curved backwall consisting of a quarter of a $20 \mathrm{~mm}$-radius circumference, the centre of which is located at a depth of $30 \mathrm{~mm}$ with respect to the front surface. Mapping of a $58^{\circ}$ arc of the backwall was simulated using parallel ultrasonic inspection beams (Figure 4(a)) and angled inspection beams (Figure 4(c)). For the parallel mapping approach, the transducer was moved with constant steps of $1 \mathrm{~mm}$ along the front surface. For the angled beam mapping approach, the transducer was translated and rotated by quantities suitable to produce longitudinal waves that strike the backwall perpendicularly at points separated by $1 \mathrm{~mm}$-long arc segments. Figures 4(a) and 4(c) illustrate, for the sake of clarity, half of the resulting ultrasonic beams. The black arrows show the propagation paths. Whereas the backwall echoes do not return to the transducer for most of the backwall points for the case in Figure 4(a), since the backwall reflects the beams in accordance with Snell's Law, the return paths always coincide with the forward paths in Figure 4(c), where incident angles are calculated considering Equation (1). The vertical dashed lines illustrate the directions along which the resulting A-scans are displayed to form the traditional B-scan perpendicular to the scanning surface and parallel to a reference direction, as defined in BS EN 1330-4:2000. This displays the B-scan (the model cross-section) on the plane through which the individual A-scans have been simulated, which contains the ultrasonic beam axes. Therefore, the resulting B-scans are shown overlaying to the part geometry in Figures 4(b) and 4(d). The B-scans are plotted with $50 \%$ transparency, to help assess their alignment with the part geometry. 


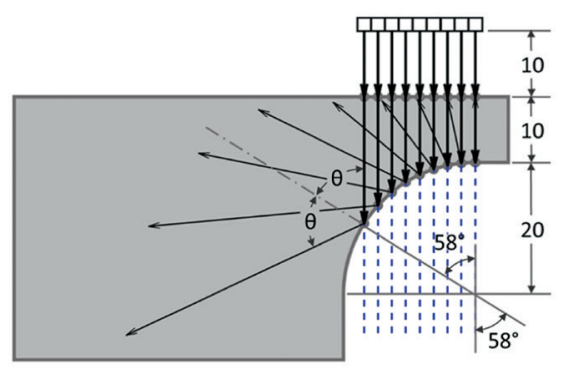

(a)

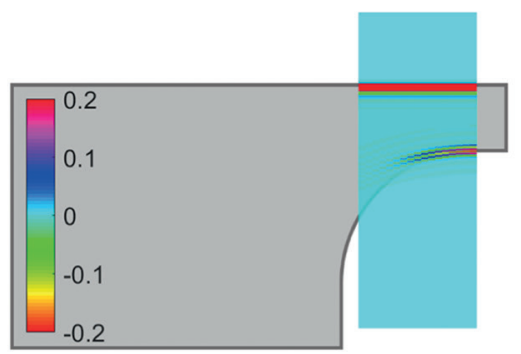

(b)

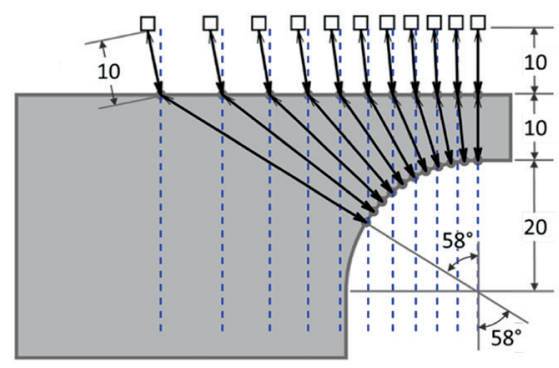

(c)

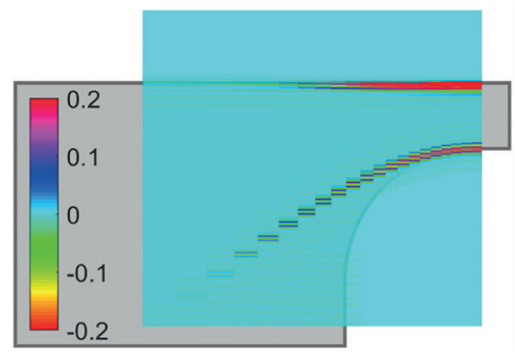

(d)

Using the corrected encoding positions to plot the $i$ th A-scan provides alignment to the originating ultrasonic beam path. By applying Equations (6) and (7) to all A-scans that form the B-scan, an undistorted version of the B-scan can be obtained (Figure 6(a)). This corrected result clearly shows the elimination of the geometric distortion.

\subsection{Intensity correction}

The amplitude (or intensity) of the backwall reflection visibly changes along the $58^{\circ}$ arc. The amplitude decreases from right to left, for both parallel beams and angled beams. Assuming unitary energy for all incidence beams, the variable amplitude is caused by the different attenuation experienced by the beams during their travel. For the case of parallel beams $\left(\theta_{1 L}=\theta_{2 L}=\theta_{2 S}=0\right)$, from Equations (4) and (5) it is possible to find that all transmitted beams $\left(L_{1}^{T}\right)$, produced at the interface between medium 1 (water) and medium 2 (aluminium), have the same intensity, since they strike the interface of

Figure 4. Ultrasonic pulse-echo with: (a) parallel beams; and (c) angled beams. (b) and (d) are the respective B-scans

The algorithm described in $^{[28]}$ has been used to triangulate the succession of A-scan points and visualise the B-scans as triangular meshes, where the colour of the points corresponds to the original amplitude of the A-scan.

The angled beams produce stronger backwall echoes, as explained above. However, the relative B-scan in Figure 4(d) is visibly distorted, since the backwall indications diverge from the reference geometry, due to the deviation between the real beam direction and the direction perpendicular to the front surface at the point of incidence. For this reason, an algorithm has been developed to resolve this geometrical distortion and improve the B-scan visualisation. For clarity in the description of such an algorithm, Figure 5 shows the original ith A-scan and the same A-scan after the correction of the distortion. The point of incidence between the $i$ th beam and the front surface is indicated by $F_{i} \equiv\left[x_{F_{i}}, y_{F_{i}}\right]$ and the encoding positions of all A-scan samples stored in the $N \times 2$ matrix are considered, $C^{i}=\left[x^{i} y^{i}\right]$, where $N$ is the number of samples. The first column $\left(x^{i}\right)$ is an $N \times 1$ vector of zeros. Since each original A-scan is displayed along the vertical direction, all points of the same A-scan share the same $x$-coordinate value, equal to $x_{F_{i}}$. The second column $\left(y^{i}\right)$ is the vector of the $y$-coordinates. This vector is the result of the manipulation of the sampling time vector $(t)$, as follows:

$$
\boldsymbol{y}^{i}=-\left[\begin{array}{c}
{\left[\boldsymbol{t}\left(1: i_{0}\right) \cdot \frac{V_{1}}{2}\right]-W} \\
{\left[\boldsymbol{t}\left(i_{0}+1: N\right) \cdot \frac{V_{2}}{2}\right]-W}
\end{array}\right] .
$$

where $i_{0}$ is the index of the last sample collected above the front surface.

Indicating with $\boldsymbol{R}_{1}^{i}$ and $\boldsymbol{R}_{2}^{i}$ the matrices of rotation around the $z$-axis (perpendicular to the $\mathrm{B}$-scan plane), respectively corresponding to the angles $\theta_{1 L}^{i}$ and $\theta_{2 L}^{i}$, the corrected matrix of encoding coordinates, $\boldsymbol{C}_{\boldsymbol{C}}^{i}$, is given by:

$\boldsymbol{C}_{\boldsymbol{C}}^{\boldsymbol{i}}=\left[\begin{array}{cc}{\left[\boldsymbol{x}^{i}\left(1: i_{0}\right)-x_{F_{i}}\right.} & \left.\boldsymbol{y}^{i}\left(1: i_{0}\right)\right] * \boldsymbol{R}_{1}^{i} \\ {\left[\boldsymbol{x}^{i}\left(i_{0}+1: N\right)-x_{F_{i}}\right.} & \left.\boldsymbol{y}^{i}\left(i_{0}+1: N\right)\right] * \boldsymbol{R}_{2}^{i}\end{array}\right]+\left[\begin{array}{ll}\boldsymbol{x}_{F_{i}} & \boldsymbol{o}\end{array}\right] \ldots$ two isotropic media with the same incidence angle. The decreasing amplitude visible in Figure 6(a) is caused by the variable incidence angle between $L_{1}^{T}$ and the backwall, between $L_{E}$ and the interface between solid and water and by the fact the return point of $L_{E}^{T}$ to the transducer surface diverges from the surface centre. This latter element alone causes a strong decay of the return wave amplitude and any a posteriori amplification is impractical, since it would produce a poor signal-to-noise ratio.

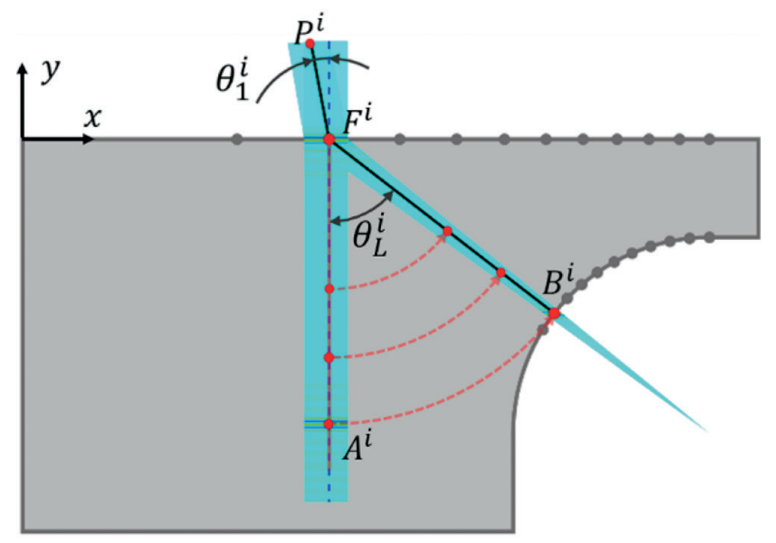

Figure 5. Correction of the ith A-scan display

Conversely, for the case of angled beams, where each incident beam has a different incidence angle and produces different longitudinal and shear refraction angles, the reflected and transmitted beams will inherit different (but predictable) energy portions. In relation to the unitary amplitude of the $i$ th incident beam, the intensity of $L_{E}^{T i}$ that comes back to the transducer is estimated using Equation (8):

$$
I^{i}=I_{12}^{T i} \cdot I_{21}^{R i} \cdot I_{21}^{T i}
$$

where $I_{12}^{T i}$ is the intensity attenuation factor of the wave transmitted from water to solid, $I_{21}^{R i}$ is the attenuation factor of the wave reflected at the backwall (the interface between material 2 and material 1) and $I_{21}^{T i}$ is the attenuation experienced by the returning echo at the 
front interface between solid and water. Figure 7 shows the plot of attenuation (in decibels) relative to such factors and the total attenuation, $I$. The attenuations are given versus the incidence angle (spanning between zero and the first critical angle, as shown on the top horizontal axis) and the refracted angle (shown on the bottom horizontal axis). The opposite of the total attenuation is used as gain to correct the intensity of the A-scans that form the B-scan.

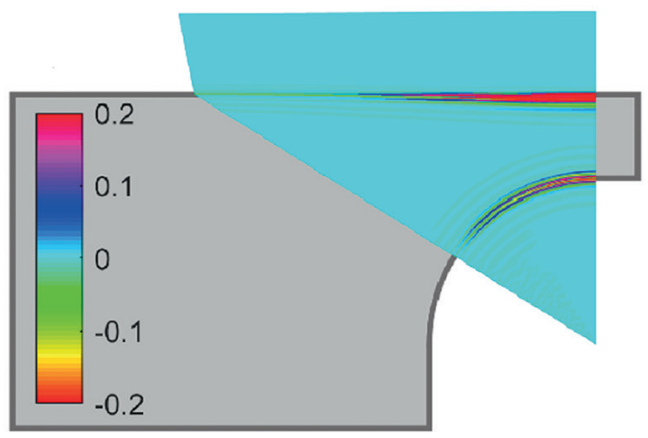

(a)

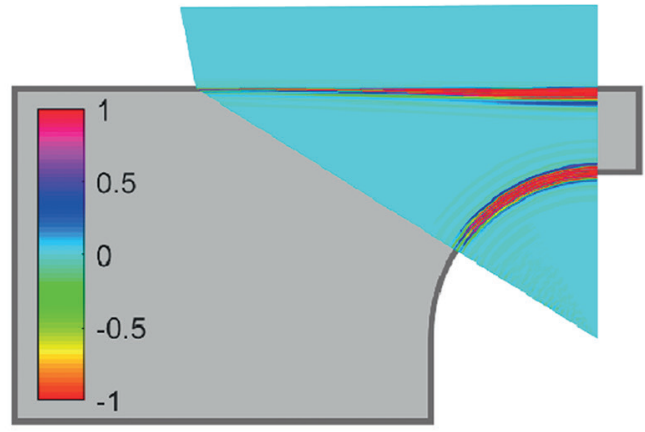

(b)

Figure 6. Correction of: (a) geometric distortion; and (b) backwall amplitude

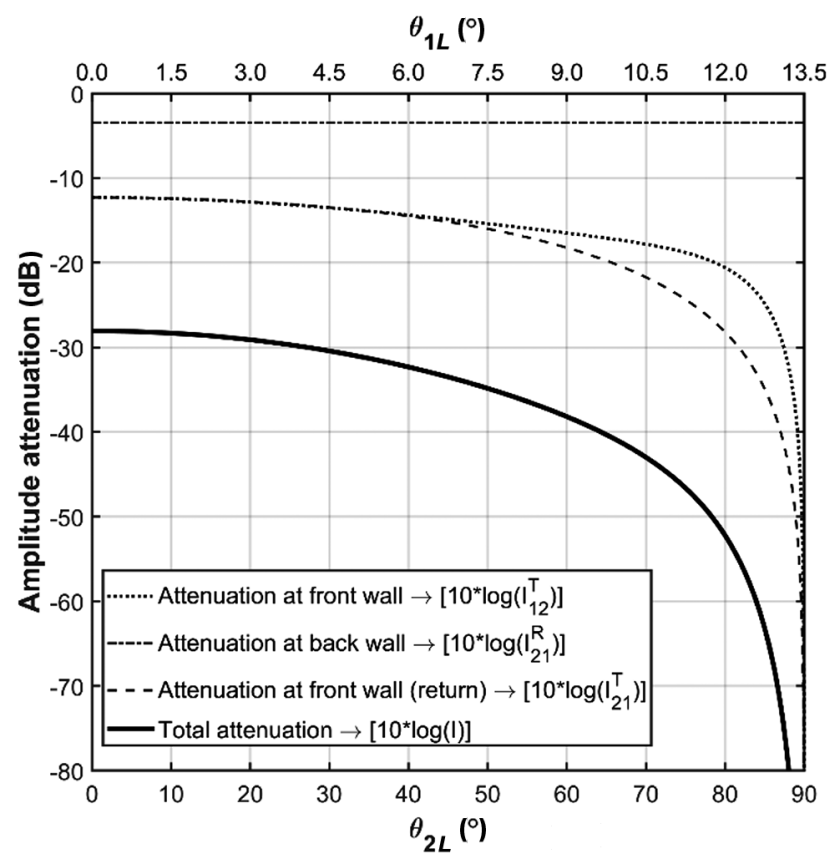

Figure 7. Plot of the attenuation factors (in decibels). The opposite value of the total attenuation is used as gain to correct the intensity of the B-scan
Therefore, the final corrected B-scan is given in Figure 6(b). The amplification of the A-scans that form the B-scan has the effect of normalising the intensity of the backwall echoes. Thus, it is important to notice that the colour bar limits have been extended from -1 to +1 in Figure $6(\mathrm{~b})$. For refracted angles smaller than $60^{\circ}$ in immersion pulse-echo inspection of aluminium samples, which is the case considered in this work, the required amplification is always smaller than $40 \mathrm{~dB}$.

\subsection{Application to a more complex backwall}

The application of the developed algorithm to a more complex backwall is now presented. The new geometry is shown in Figure 8.

The sample model has a flat front surface and a curved backwall consisting of two $60^{\circ}$ arcs of a $20 \mathrm{~mm}$-radius circumference, separated by a $120^{\circ}$ arc of a circumference with the same radius but opposite curvature. This geometry more closely resembles the backwall profile of a stiffening stringer in aerospace structures. A $2 \mathrm{~mm}$-radius diameter side-drilled hole (SDH) was included, located $30 \mathrm{~mm}$ from the left-hand corner of the model and at a depth of $20 \mathrm{~mm}$. The same aluminium material was considered, having the acoustic properties defined above. The same probe was also considered, simulating the ultrasonic wave propagation through CIVA. Mapping of the backwall was simulated using the standard parallel ultrasonic inspection beams (Figure 8(a)) and the angled inspection beams (Figure 8(c)). For the parallel mapping approach, the transducer was moved with constant steps of $1 \mathrm{~mm}$ along the front surface. Although this approach obtains a clear echo from the SDH, the backwall is almost not mapped. For the angled beam mapping approach, explained above, the transducer was translated and rotated by quantities suitable to produce longitudinal waves that were perpendicularly incident on the backwall at points separated by $1 \mathrm{~mm}$-long arc segments. The incident angles were appropriately calculated, according to Snell's Law, through Equation (1). In Figure 8(d), the mismatch between the B-scan data and the geometry of the sample is evident. The reflection wave from the simulated SDH is dislocated from the position of the modelled defect. Applying the introduced approach for the correction of geometry and amplitude distortion, the resulting B-scans in Figures 9(a) and 9(b) are obtained. The two anomalies in the backwall echo are caused by the interference of the front surface secondary echo, which distorts the reflection from the backwall. The echo from the $2 \mathrm{~mm}$-diameter SDH is correctly scaled and positioned.

\section{Conclusion and future work}

Ultrasonic imaging of complex backwalls is currently challenging. This work has introduced a novel approach to improve the ultrasonic inspectability of parts with complex backwalls. Conventional approaches for ultrasonic inspection of such parts require separate inspections of both surfaces. Using ultrasonic phased array probes and state-of-the-art instrumentation allows ultrasonic energy to be sent into a part at wide ranges of focusing depths and steering angles. This allows for tracking of the backwall profile, thus hitting it at normal incidence and maximising the amplitude of the reflected echo at any point. This work has shown that a cross-sectional scan resulting from multiple ultrasonic beams, which are sent at variable incidence angles, presents significant geometrical distortion and is of limited use for accurate defect visualisation and sizing. This paper introduced a generalised algorithm able to remove geometric distortions and the effect that variable attenuation factors have on the received amplitudes. Future work will extend the approach 
(currently for isotropic materials) to consider anisotropic composite materials. The ultimate goal is to make it possible to map complex backwalls from the frontwall side (a typical application being the inspection of in-service components, where access from only a single side is usually possible). The correction algorithm will also be extended to B-scan correction for focused ultrasonic beams produced by concave-surface single-element probes and phased array probes.

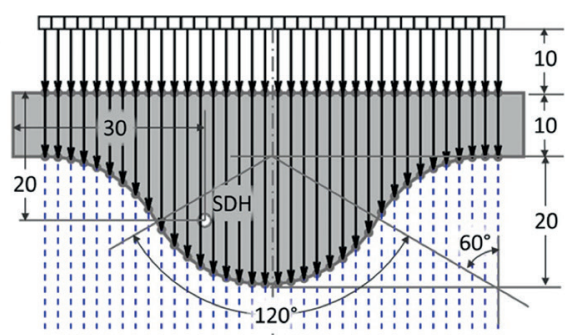

(a)

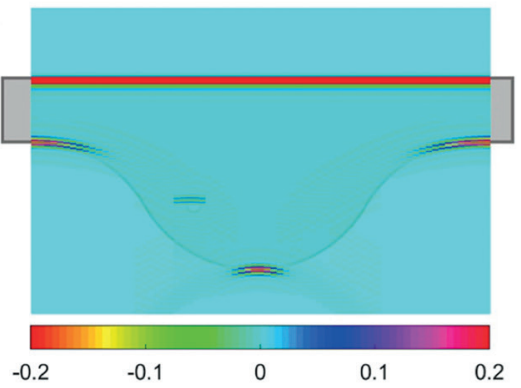

(b)

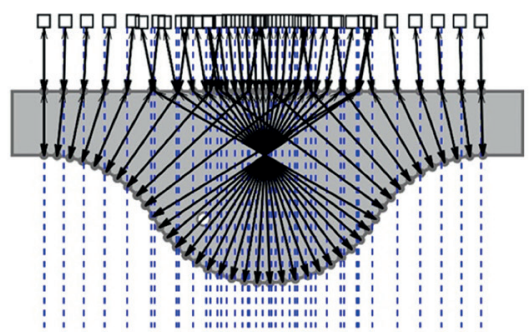

(c)

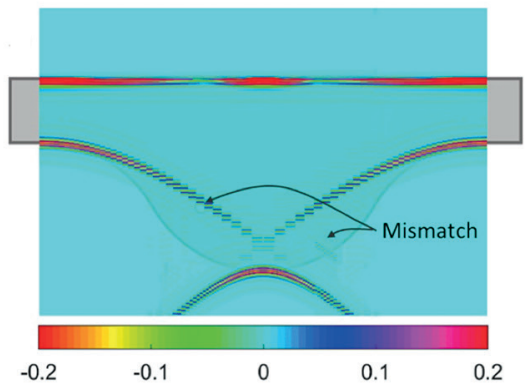

(d)

\section{Acknowledgements}

This work was funded by Spirit AeroSystems Ltd (Prestwick, UK). The authors also acknowledge additional support received from the Autonomous Inspection in Manufacturing and Remanufacturing (AIMaReM) project, funded by the UK Engineering and Physical Sciences Research Council (EPSRC) through grant EP/N018427/1.

\section{References}

1. T Kundu, Ultrasonic Nondestructive Evaluation, CRC Press, 2003.

2. R Huang, 'Ultrasonic modelling for complex geometries and materials', Engineering Mechanics, Iowa State University, Ames, Iowa, USA, 2006.

3. G A Deschamps, 'Ray techniques in electromagnetics', Proceedings of IEEE, Vol 60, pp 1022-1035, 1972.

4. C Holmes, B W Drinkwater and P D Wilcox, 'Post-processing of the full matrix of ultrasonic transmit-receive array data for non-destructive evaluation',

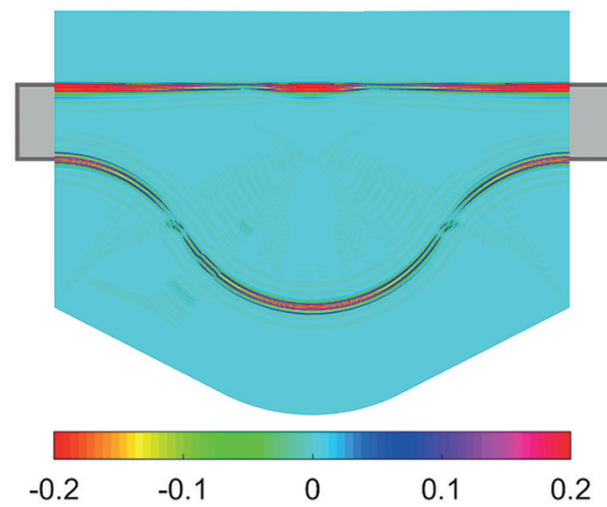

(a)

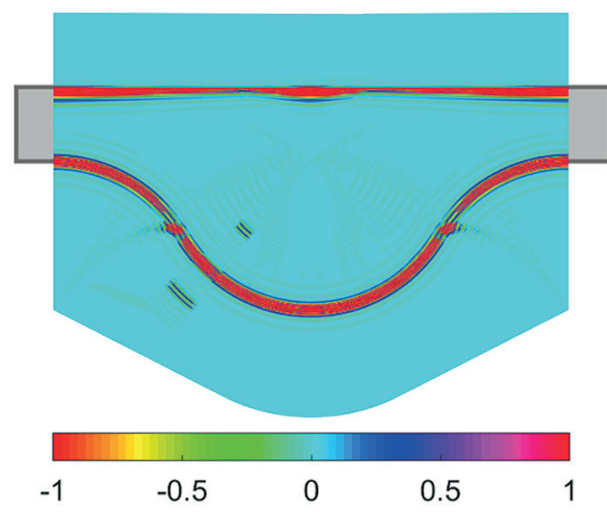

(b)

Figure 9. Correction of: (a) geometric distortion; and (b) backwall amplitude for a model with a complex backwall and side-drilled hole
NDT\&E International, Vol 38, No 8, pp 701-711, 2005.

5. M Sutcliffe, M Weston, P Charlton, K Donne, B Wright and I Cooper, 'Full matrix capture with time-efficient auto-focusing of unknown geometry through dual-layered media', Insight: Non-Destructive Testing and Condition Monitoring, Vol 55, No 6, pp 297-301, 2013.

6. M Parrilla, A Ibáñez, J Camacho and C Fritsch, 'Fast focal law computing for non-destructive testing with phased arrays', Proceedings of the 2007 International Congress on Ultrasonics (ICU), Vienna, Austria, pp 1-4, 9-11 April 2007.

7. A J Hunter, B W Drinkwater and P D Wilcox, 'Autofocusing ultrasonic imagery for non-destructive testing and evaluation of specimens with complicated geometries, NDT\&E International, Vol 43, No 2, pp 78-85, 2010.

8. S Chatillon, S Mahaut and P Dubois, 'Simulation of advanced UT phased array techniques with matrix probes and dynamic settings for complex component inspections', AIP Conference Proceedings, Vol 1096, No 1, p 864, 2009.

9. E Hoyle, M Sutcliffe, P Charlton and J Rees, 'Virtual source aperture imaging with auto-focusing of unknown complex geometry through dual-layered media', Proceedings of the 56th Annual British Conference on Non-Destructive Testing (NDT 2017), Telford, UK, 5-7 September 2017.

10. C N MacLeod, S G Pierce, M Morozov, R Summan, G Dobie, P McCubbin et al, Automated metrology and NDE measurements for increased throughput in aerospace component manufacture', 41st Annual Review of Progress in Quantitative Non-Destructive Evaluation, pp 1958-1966, 2015.

11. T Sattar, 'Robotic non-destructive testing', Industrial Robot: An International Journal, Vol 37, No 5, 2010.

12. D L Hopkins, M Brassard, G A Neau, J-N Noiret, W V Johnson and L L Ber, 'Surface-adaptive ultrasound (SAUL) for phased 
array inspection of composite specimens with curved edges and complex geometry', AIP Conference Proceedings, Vol 1511, No 1, pp 809-816, 2013.

13. S Robert, O Casula, O Roy and G Neau, 'Real-time inspection of complex composite structures with a self-adaptive ultrasonic technique', Proceedings of the 18th World Conference on NonDestructive Testing (WCNDT 2012), Durban, South Africa, 16-20 April 2012.

14. F Bentouhami, B Campagne, E Cuevas, T Drake, M Dubois, T Fraslin et al, 'LUCIE - a flexible and powerful laser ultrasonic system for inspection of large CFRP components', Proceedings of the 2nd International Symposium on Laser Ultrasonics Science, Technology and Applications, Talence, France, 5-8 July 2010.

15. A Maurer, W D Odorico, R Huber and T Laffont, 'Aerospace composite testing solutions using industrial robots', Proceedings of the 18th World Conference on Non-Destructive Testing (WCNDT 2012), Durban, South Africa, 16-20 April 2012.

16. J T Stetson and W D Odorico, 'Robotic inspection of fibrereinforced aerospace composites using phased array UT', Proceedings of the 40th Annual Review of Progress in Quantitative NDE, Baltimore, Maryland, USA, 21-26 July 2013.

17. C Mineo, S Pierce, B Wright, I Cooper and P Nicholson, 'PAUT inspection of complex-shaped composite materials through six DOFs robotic manipulators', Insight: Non-Destructive Testing and Condition Monitoring, Vol 57, No 3, pp 161-166, 2015.

18. E Cuevas, S Hernandez and E Cabellos, 'Robot-based solutions for NDT inspections: integration of laser ultrasonics and aircoupled ultrasounds for aeronautical components', Proceedings of the 25th ASNT Research Symposium, New Orleans, Louisiana, USA, pp 39-46, 11-14 April 2016.

19. R A Smith, 'Use of $3 \mathrm{D}$ ultrasound datasets to map the localised properties of fibre-reinforced composites', $\mathrm{PhD}$ thesis, University of Nottingham, 2010.

20. C Mineo, S G Pierce, P I Nicholson and I Cooper, 'Robotic path planning for non-destructive testing - a custom MATLAB toolbox approach', Robotics and Computer-Integrated Manufacturing, Vol 37, pp 1-12, 2016.

21. M Brassard, D L Hopkins and J N Noiret, 'Integration of robotics and surface-adaptive phased array UT to achieve fully automated inspection of complex composite parts', Proceedings of ASNT NDT of Composites Conference, Seattle, Washington, USA, 13-14 May 2013.

22. C Mineo, C MacLeod, M Morozov, S G Pierce, T Lardner, $\mathrm{R}$ Summan et al, 'Fast ultrasonic phased array inspection of complex geometries delivered through robotic manipulators and high-speed data acquisition instrumentation', Proceedings of the 2016 IEEE International Ultrasonics Symposium (IUS), Tours, France, pp 1-4, 18-21 September 2016.

23. C Mineo, C MacLeod, R Su, D Lines, S Davì, B Cowan et al, 'Robotic geometric and volumetric inspection of high-value and large-scale aircraft wings', Proceedings of the 2019 IEEE International Workshop on Metrology for AeroSpace, Torino, Italy, 19-21 July 2019.

24. R Su, C Mineo, C N MacLeod, S G Pierce and A Gachagan, 'Multi-aperture beamforming for automated large structure inspection using ultrasonic phased arrays', AIP Conference
Proceedings, Vol 2102, No 1, p 100009, 2019.

25. J Zhang, B W Drinkwater and P D Wilcox, 'Efficient immersion imaging of components with non-planar surfaces', IEEE Transactions on Ultrasonics, Ferroelectrics, and Frequency Control, Vol 61, No 8, pp 1284-1295, 2014.

26. D Hopkins, $M$ Datuin and $M$ Brassard, 'Challenges and solutions for ultrasonic phased array inspection of polymermatrix composites at production rates', AIP Conference Proceedings, Vol 2102, No 1, p 100002, 2019.

27. A Maurer, W Deodorico, R Huber and T Laffont, 'Aerospace composite testing solutions using industrial robots', Proceedings of the 18th World Conference on Non-Destructive Testing (WCNDT 2012), Durban, South Africa, 16-20 April 2012.

28. C Mineo, J Riise, R Summan, C N MacLeod and S G Pierce, 'Index-based triangulation method for efficient generation of large three-dimensional ultrasonic C-scans', Insight: NonDestructive Testing and Condition Monitoring, Vol 60, No 4, pp 183-189, 2018.

29. J Camacho, J F Cruza, J Brizuela and C Fritsch, 'Automatic dynamic depth focusing for NDT', IEEE Transactions on Ultrasonics, Ferroelectrics, and Frequency Control, Vol 61, No 4, pp 673-684, April 2014.

30. J F Cruza, J Camacho, J M Moreno and C Fritsch, 'Ultrafast hardware-based focal law calculator for automatic focusing, NDT\&E International, Vol 74, pp 1-7, 2015.

31. O Casula, C Poidevin, G Cattiaux and P Dumas, 'Control of complex components with smart flexible phased arrays', AIP Conference Proceedings, Vol 820, No 1, pp 829-836, 2006.

32. J M Davis and M Moles, 'Resolving capabilities of phased array sectorial scans (S-scans) on diffracted tip signals' Insight: NonDestructive Testing and Condition Monitoring, Vol 48, No 4, pp 233-239, 2006.

33. J Camacho, J F Cruza, J Brizuela and C Fritsch, 'Automatic dynamic depth focusing for NDT', IEEE Transactions on Ultrasonics, Ferroelectrics, and Frequency Control, Vol 61, No 4, pp 673-684, April 2014.

34. D Cassereau, 'Focusing with ultrasonic phased arrays comparison between time delay laws and time reversal, Proceedings of the 2000 IEEE International Conference on Phased Array Systems and Technology, Dana Point, California, USA, 21-25 May 2000.

35. J F Cruza, J Camacho, L Serrano-Iribarnegaray and C Fritsch, 'New method for real-time dynamic focusing through interfaces,' IEEE Transactions on Ultrasonics, Ferroelectrics, and Frequency Control, Vol 60, No 4, pp 739-751, 2013.

36. J Habermehl, 'PA probe and radius wedges for composite inspection', Olympus Internal Technical Report, February 2009.

37. Olympus, 'Introduction to phased array ultrasonic technology applications', Available at: www.olympus-ims.com/en/books/ $\mathrm{pa} / \mathrm{pa}$-intro

38. B A Auld, Acoustic Fields and Waves in Solids, Vol 2, John Wiley \& Sons, 1973.

39. J D N Cheeke, Fundamentals and Applications of Ultrasonic Waves, 2nd edition, 2012.

40. P Calmon, S Mahaut, S Chatillon and R Raillon, 'CIVA: an expertise platform for simulation and processing NDT data', Ultrasonics, Vol 44, pp e975-e979, 2006. 